\title{
Cardiovascular Incidence in 6900 Patients with Differentiated Thyroid Cancer: a Swedish Nationwide Study
}

\author{
Maximilian Zoltek $^{1} \cdot$ Therese M.-L. Andersson $^{2} \cdot$ Christel Hedman $^{1,3}$ - Catharina Ihre- \\ Lundgren $^{1,4} \cdot$ Caroline Nordenvall ${ }^{1,5}$
}

\begin{abstract}
Introduction To minimize recurrence risk in differentiated thyroid cancer (DTC), TSH is usually lifelong suppressed with levothyroxine. A common consequence of this treatment is subclinical hyperthyroidism which can induce cardiovascular disease (CV). This study's aim was to compare CV incidence in DTC patients with the general population in Sweden.

Materials and methods All Swedish patients diagnosed with DTC in 1987-2013 were included in the cohort study. Lifelong TSH suppression treatment was assumed to be administered to patients in compliance with prevalent national guidelines. Patients were followed from 1 year after DTC diagnosis until December 31, 2014, death, or migration. The event of interest was hospitalization due to any of the following diseases: atrial fibrillation (AF), cerebrovascular disease, cerebral infarction, ischemic heart disease, ischemic heart attack, and heart failure. Standardized incidence ratios (SIRs) were calculated to compare CV incidence between DTC patients and the general population.

Results The cohort consisted of 6900 patients with DTC. Hospitalization was increased among DTC patients for AF (SIR 1.66, CI 95\% 1.41-1.94), and women faced increased hospitalization for cerebrovascular disease (SIR 1.20 CI 95\% 1.04-1.38). Regarding the remaining CV diseases, no consistent difference in SIR between the groups was observed.

Conclusion Compared to the general population, DTC patients have a higher incidence in AF, and female face a slightly higher incidence in cerebrovascular disease. However, there was no difference in hospitalization for other studied CV diseases between DTC patients and the general population.
\end{abstract}

This paper was presented as an oral presentation at the IAES meeting/48th World Congress of Surgery August 11-15, 2019 in Kraków, Poland.

Maximilian Zoltek

maximilian.zoltek@ki.se

Therese M.-L. Andersson

therese.m-l.andersson@ki.se

Christel Hedman

christel.hedman@ki.se

Catharina Ihre-Lundgren

cia.ihre-lundgren@ki.se
Caroline Nordenvall

caroline.nordenvall@ki.se

1 Department of Molecular Medicine and Surgery, Karolinska Institutet, Stockholm, Sweden

2 Department of Medical Epidemiology and Biostatistics, Karolinska Institutet, Stockholm, Sweden

3 Stockholms Sjukhem Foundation's R\&D Department, Stockholm, Sweden 


\section{Introduction}

Differentiated thyroid cancer (DTC) accounts for more than $95 \%$ cases of thyroid carcinomas [1], and patients face a good prognosis with a 10-year relative survival exceeding $90 \%$ [2]. Curative treatment consists of total thyroidectomy and radioactive iodine treatment (RAI) [3]. Since thyrotropin (TSH) stimulates cancer cells [1, 3], patients usually receive levothyroxine in doses to suppress TSH, and it is known that a more aggressive suppression therapy reduces recurrence risk [4]. More, recent guidelines recommend individualized long-term levels of TSH suppression, considering not only the aggressiveness of the cancer disease, but also side effects of the treatment [5].

A known adverse effect of TSH suppression is subclinical hyperthyroidism, which is well studied in patients without DTC, being an established risk factor of atrial fibrillation (AF) and heart failure (HF) [6, 7]. Physiological effects include an increase in the heart rate and left ventricular size $[8,9]$, decreased arterial elasticity, and negative prothrombotic effects [10-14]. More, an inflammatory process of the carotid wall has also been suggested to play a role in the pathophysiological process [15].

Since expected survival in DTC is good, patients have risk to incur illnesses from the treatment rather than dying from cancer itself [16]. Currently, there are small- and medium-scale studies on cardiovascular disease in DTC patients with varying methodological approaches. Some publications report increased incidence in AF [10, 17, 18]; however, no relationship between suppressed TSH levels and cardiovascular disease has consistently been proven.

We have previously, in a national cohort study of 6900 patients, reported that DTC patients face an increased risk of mortality related to AF compared to the general population [19]. In this study, we intend to pursue the ambition of clarifying the risk of cardiovascular disease in DTC patients and hypothesize that patients are at higher risk of cardiovascular morbidity compared to the general population.

\section{Materials and methods}

In Sweden, the healthcare system is publicly funded, and each resident is assigned a unique personal identity number [20], which allows the National Board of Health and

4 Department of Breast, Endocrine Tumours and Sarcoma, D2:02, Karolinska University Hospital, Stockholm, Sweden

5 Center for Digestive Disease, Division of Coloproctology, D2:05, Karolinska University Hospital, Stockholm, Sweden
Welfare to run nationwide registers of excellent quality containing information on hospitalizations and cancer disease, as well as complete follow-up with information on migration and cause of mortality [21]. From the Swedish Cancer Registry in 1987-2013 DTC, International Classification of Disease (ICD) 7194, pathology-anatomy diagnosis 096 was identified. During the study period, the standard surgical intervention was total thyroidectomy according to national guidelines, and only the primary thyroid cancer diagnosis was considered in individuals with multiple diagnoses. Data on cardiovascular disease for DTC patients, as well as the general population, were retrieved from the Inpatient Registry, and date of death, as well as mortality cause, was collected from the Cause of Death Registry. Patients that remained in the cohort for less than 1 year after diagnosis were excluded. Lifelong TSH suppression was assumed to be administered in compliance with prevalent guidelines during the study period.

\section{Endpoints}

To study potential cardiovascular effects in DTC patients related to the postoperative treatment, patients were followed from 1 year after DTC diagnosis until the last day of 2014, death or migration. The endpoint of primary interest was hospitalization for $\mathrm{CV}$ disease, and multiple events per patient were allowed. Standardized incidence ratios (SIRs) were calculated to compare CV incidence in DTC patients and the general population. We investigated six different $\mathrm{CV}$ endpoints and categorized them by the primary diagnosis at hospital discharge: ischemic heart disease (ICD9:410-414 ICD-10: I20-25), ischemic heart attack (ICD9:410 ICD-10 1:I21, I22), heart failure (ICD-9:428 ICD10:I50), cerebral infarction (ICD-9:431, 434, 436 ICD-10: I61, I63, I64), cerebrovascular disease (ICD-9:430-434, 436-438 ICD-10:I60-69), and atrial fibrillation (ICD9:427D, 427A ICD-10: I48).

\section{Covariates}

The study population was subdivided by age at diagnosis, calendar period at diagnosis, and sex. For patients included in 2005 or later, TNM classification was available.

\section{Statistical analyses}

Standardized incidence ratios (SIRs) were calculated to compare CV incidence between DTC patients and the general population for cardiovascular endpoints listed above. SIRs were calculated by gender, age, and calendar period (both in 1-year strata). In categories given above, SIRs and accompanying 95\% confidence intervals (CI) were computed. All aforementioned SIRs were computed 
for individual $\mathrm{CV}$ primary endpoints. Further, cumulative incidence considering competing risks was calculated for cardiovascular endpoints by age, sex, and year. Unlike SIRs, only first cardiovascular event, for each endpoint, was taken into consideration when computing cumulative incidences.

STATA 12, Texas, USA, was employed for analyses. Ethical consent was obtained from the Ethical Board at Karolinska Institutet (Stockholm, Sweden), Dnr: 2014/71431.

\section{Results}

The cohort consisted of 6900 patients who were diagnosed with DTC from 1987 to 2013 and approximately 3/4 of them were women (Table 1). More than half of the patients

Table 1 Descriptive statistics of 6900 Swedish DTC patients diagnosed in 1987-2013

\begin{tabular}{|c|c|c|}
\hline \multicolumn{3}{|l|}{ Entire cohort } \\
\hline & $N$ & $\%$ \\
\hline All & 6900 & 100 \\
\hline \multicolumn{3}{|l|}{ Sex } \\
\hline Male & 1812 & 26.26 \\
\hline Female & 5088 & 73.74 \\
\hline \multicolumn{3}{|c|}{ Age at inclusion } \\
\hline$<45$ & 2523 & 36.57 \\
\hline $45-54$ & 1338 & 19.39 \\
\hline $55-64$ & 1161 & 16.83 \\
\hline $65-74$ & 1062 & 15.39 \\
\hline$\geq 75$ & 816 & 11.83 \\
\hline \multicolumn{3}{|c|}{ Year of inclusion } \\
\hline $1987-1995$ & 1941 & 28.12 \\
\hline 1996-2004 & 1992 & 28.87 \\
\hline 2005-2013 & 2967 & 43.00 \\
\hline \multicolumn{3}{|c|}{ TNM $($ diagnosed $\geq 2005)$} \\
\hline \multicolumn{3}{|l|}{ T-stage } \\
\hline Missing & 668 & 22.51 \\
\hline 0 & 28 & 0.94 \\
\hline I & 1085 & 36.57 \\
\hline II & 634 & 21.37 \\
\hline III & 408 & 13.75 \\
\hline IV & 144 & 4.85 \\
\hline \multicolumn{3}{|l|}{$\mathrm{N}$-stage } \\
\hline Missing & 1118 & 37.68 \\
\hline 0 & 1262 & 42.53 \\
\hline I & 587 & 19.78 \\
\hline
\end{tabular}

were 54 years old or younger at diagnosis, and the mean follow-up time was 9.66 years.

Figure 1 depicts SIRs for the six different CV endpoints. Patients had increased hospitalization for AF (SIR 1.66 CI 95\% 1.41-1.94), as well as cerebrovascular disease (SIR 1.14 CI 95\% 1.01-1.29) which on a subgroup analysis was associated with women (SIR 1.20 CI 95\% 1.04-1.38). For the remaining four endpoints (cerebral infarction, ischemic heart attack, ischemic heart disease, heart failure), there was no difference in hospitalization among DTC patients compared with the general population (Fig. 1).

\section{Atrial fibrillation}

There were in total 542 events of AF hospitalization, which pertained to 279 unique individuals. Both men and women on a standalone basis had elevated risk ratios of AF. Further, SIRs were elevated among all age categories except in patients $\geq 75$ years of age, and SIRs decreased with age at diagnosis and with follow-up duration. More, incidence increased with TNM classifications and was consistent across calendar periods (Table 2).

\section{Cumulative incidence}

Cumulative incidence in atrial fibrillation in total, as well as subdivided by sex, is presented as complementary data, by Figs. 2, 3 and 4 .

\section{Discussion}

In this nationwide cohort study of 6900 patients, we have found DTC patients to be at significantly higher risk of hospitalization for AF compared to the general population. Elevated risk rates of $\mathrm{AF}$ affect both sexes, where men seem to have a slightly higher SIR than their female counterparts.

In addition to gender, follow-up time and age at diagnosis were associated with an elevated SIR of AF hospitalization. During the first five follow-up years, patients run a twofold increased rate of AF hospitalization. While sex and age at diagnosis are heterogeneous traits among patients, more than $90 \%$ survive the first 10 years postcancer diagnosis [1], which implies that the vast majority of DTC patients will face a higher risk rate of AF the first years after diagnosis. More, patients 54 years or younger at diagnosis were associated with an approximately 2-2.5 times elevated risk rate of $\mathrm{AF}$. This is also clinically meaningful to bear in mind, since more than half of the study population belonged to this particular age category.

We have also noted that female DTC patients run a slightly elevated rate of cerebrovascular disease compared 


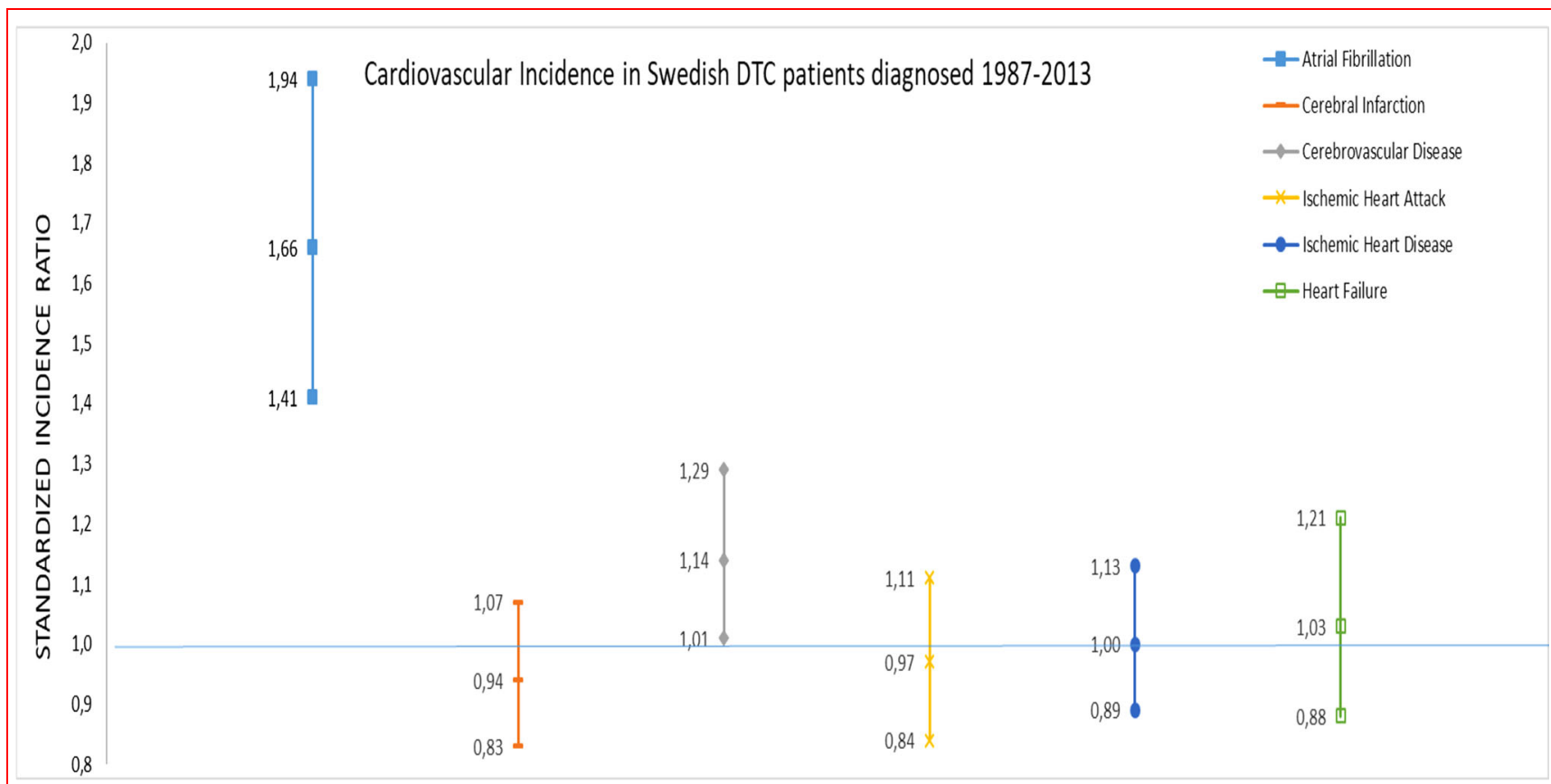

Fig. 1 Cardiovascular incidence, displayed as standardized incidence ratios (SIR) and 95\% confidence intervals subdivided by six cardiovascular endpoints. The blue line crossing SIR 1.0 equals the incidence rate of the general population

Table 2 Descriptive statistics and standardized incidence ratios (SIRs) and 95\% confidence intervals for atrial fibrillation hospitalization

\begin{tabular}{|c|c|c|c|c|}
\hline \multirow[t]{2}{*}{ ICD subgroup } & \multirow[t]{2}{*}{ \# Incidental events atrial fibrillation } & \multirow[t]{2}{*}{ SIR } & \multicolumn{2}{|c|}{$95 \% \mathrm{CI}$} \\
\hline & & & Lower & Upper \\
\hline All & 542 & 1.66 & 1.41 & 1.94 \\
\hline \multicolumn{5}{|l|}{ Sex } \\
\hline Male & 186 & 1.80 & 1.33 & 2.43 \\
\hline Female & 356 & 1.59 & 1.32 & 1.91 \\
\hline \multicolumn{5}{|c|}{ Age at diagnosis } \\
\hline$<45$ & 63 & 2.67 & 1.61 & 4.41 \\
\hline $45-54$ & 101 & 2.02 & 1.40 & 2.94 \\
\hline $55-64$ & 166 & 2.01 & 1.44 & 2.82 \\
\hline $65-74$ & 140 & 1.38 & 1.07 & 1.79 \\
\hline$\geq 75$ & 72 & 1.03 & 0.75 & 1.41 \\
\hline \multicolumn{5}{|l|}{ Period } \\
\hline 1987-1995 & 278 & 1.60 & 1.30 & 1.97 \\
\hline 1996-2004 & 188 & 1.70 & 1.25 & 2.30 \\
\hline 2005-2013 & 76 & 1.77 & 1.23 & 2.55 \\
\hline \multicolumn{5}{|c|}{ Follow-up (years) } \\
\hline $0-5$ & 227 & 2.00 & 1.63 & 2.46 \\
\hline $5-10$ & 149 & 1.69 & 1.29 & 2.21 \\
\hline $10-15$ & 79 & 1.23 & 0.91 & 1.66 \\
\hline $15-20$ & 66 & 1.57 & 1.07 & 2.29 \\
\hline $20-25$ & 21 & 1.13 & 0.53 & 2.42 \\
\hline
\end{tabular}




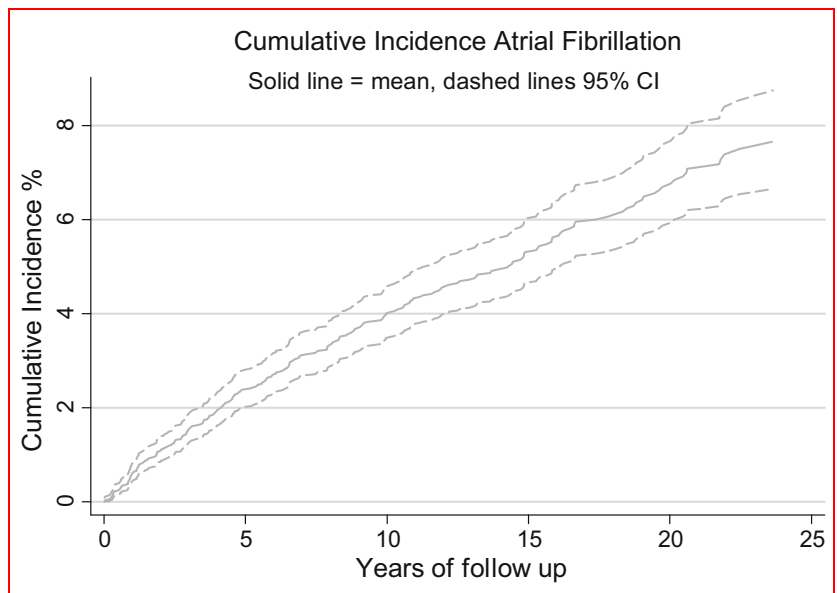

Fig. 2 Cumulative incidence of atrial fibrillation in all Swedish patients diagnosed with DTC in 1987-2013 (mean with 95\% CI)

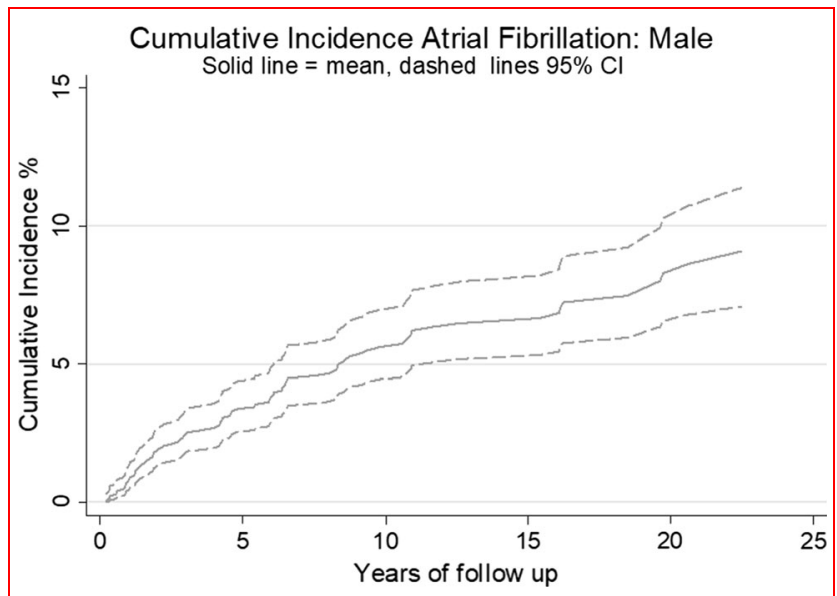

Fig. 3 Cumulative incidence in atrial fibrillation in all Swedish men diagnosed with DTC in 1987-2013 (mean with 95\% CI)

to the general population, but not regarding cerebral infarction. Concerning critical cardiac conditions such as ischemic heart disease, ischemic heart attack, or heart failure, we did not notice DTC patients to be at different risk than the general population.

Our results are in line with recent publications, indicating an elevated incidence in AF among DTC patients. A retrospective cohort study of 901 patients [18] found DTC patients to have a higher incidence in $\mathrm{AF}$, and in a subgroup analysis linked low TSH values with increased AF morbidity. Another case-control study of roughly 500 patients [17] likewise reported higher incidence in AF among DTC patients, but instead of TSH levels found AF incidence to correlate with radioactive iodine treatment. We stay assured that these potential explanatory variables of increased AF morbidity presented in the previous literature have been fixed over time in this study and that patients have received care according to The National Swedish Clinical Guidelines. The guidelines stipulate radioactive iodine and TSH suppression treatment to all DTC patients, and this assumption is further strengthened by unpublished data from a recent review of 300 patients from the Stockholm area 1995-2013, in where all have been treated according to the prevalent guidelines undergoing total thyroidectomy, receiving radioactive iodine treatment and being included in the TSH suppression program. Further strengths of this study come with the vast patient material. To our knowledge, no previous study has described cardiovascular morbidity in a whole nation during a follow-up period extending 20 years. More, the national registries are externally validated presenting excellent levels of quality [21].

While constituting a strength in describing the cardiovascular disease panorama, the registry approach poses simultaneously the main weakness of this study since it has not been feasible to collect information on TSH levels, radioactive iodine treatment, and other patient characteristics for several thousands of patients, trying to explain differences in cardiovascular morbidity between groups. More, we have only investigated cases of cardiovascular inward care, but due to drawbacks in outpatient registries not included patients that have been treated for cardiovascular disease in the specialist outward or primary care. This approach comes with the strength of presenting reliable data on a large scale regarding inward care, at the cost of not being able to depict the total cardiovascular disease panorama. However, to our knowledge, there is no published data that have shown DTC patients to be at considerable cardiovascular risk at diagnosis. This is further supported by the earlier mentioned unpublished data from the Stockholm area 1995-2013, where the vast majority of the 300 patients had neither a cardiovascular disease nor a cardiovascular comorbidity prior to surgery.

\section{Conclusions}

We have in 6900 DTC patients over a 20-year study period shown that DTC patients were hospitalized to a larger extent for AF compared with the general population. Further, young DTC patients relatively display the highest increase in rate regarding AF hospitalization. However, except for the case of cerebrovascular disease in female, we could not find DTC patients to be hospitalized for other cardiovascular diseases such as cerebral infarction, ischemic heart disease, ischemic heart attack, or heart failure to a higher rate than the general population. This study, alongside with the recent previous literature, raises 


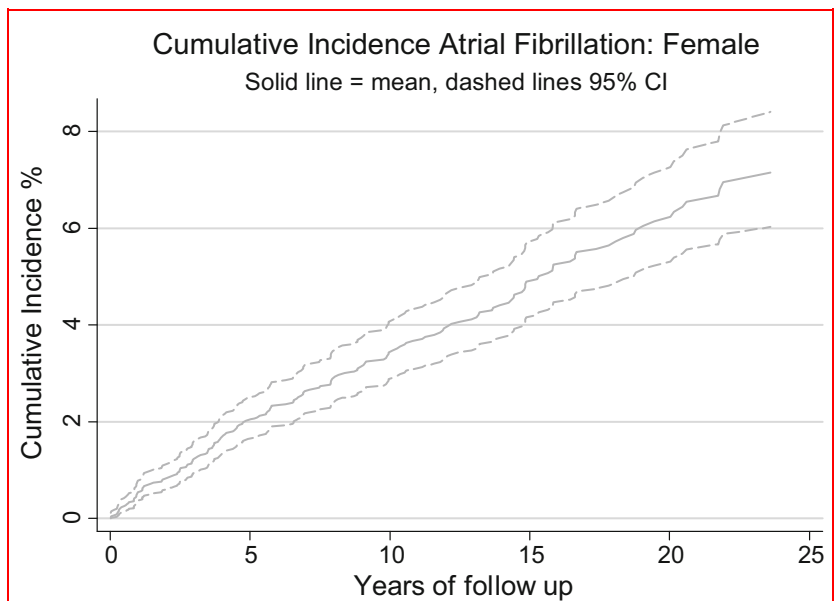

Fig. 4 Cumulative incidence in atrial fibrillation in all Swedish females diagnosed with DTC in 1987-2013 (mean with 95\% CI)

the need for further research on why DTC patients face higher risks of AF disease.

Acknowledgements Open access funding provided by Karolinska Institute.

Funding The study was supported by grants from Kirurgisk Vetenskap, Grönbergs Stiftelse, and Svenska Läkaresällskapet.

\section{Compliance with ethical standards}

Conflict of interest The authors declare that they have no competing interests.

Open Access This article is distributed under the terms of the Creative Commons Attribution 4.0 International License (http://crea tivecommons.org/licenses/by/4.0/), which permits unrestricted use, distribution, and reproduction in any medium, provided you give appropriate credit to the original author(s) and the source, provide a link to the Creative Commons license, and indicate if changes were made.

\section{References}

1. Cabanillas ME, McFadden DG, Durante C (2016) Thyroid cancer. Lancet 388:2783-2795

2. Lundgren CI, Hall P, Ekbom A et al (2003) Incidence and survival of Swedish patients with differentiated thyroid cancer. Int $\mathbf{J}$ Cancer 106:569-573

3. Hundahl SA, Fleming ID, Fremgen AM et al (1998) A National Cancer Data Base report on 53,856 cases of thyroid carcinoma treated in the U.S., 1985-1995 [see commetns]. Cancer 83:2638-2648

4. Pujol P, Daures JP, Nsakala N et al (1996) Degree of thyrotropin suppression as a prognostic determinant in differentiated thyroid cancer. J Clin Endocrinol Metab 81:4318-4323
5. Haugen BR, Alexander EK, Bible KC et al (2016) 2015 American Thyroid Association Management Guidelines for adult patients with thyroid nodules and differentiated thyroid cancer: the American Thyroid Association Guidelines Task Force on thyroid nodules and differentiated thyroid cancer. Thyroid 26:1-133

6. Collet TH, Gussekloo J, Bauer DC et al (2012) Subclinical hyperthyroidism and the risk of coronary heart disease and mortality. Arch Intern Med 172:799-809

7. Biondi B, Kahaly GJ (2010) Cardiovascular involvement in patients with different causes of hyperthyroidism. Nat Rev Endocrinol 6:431-443

8. Biondi B, Palmieri EA, Lombardi G et al (2002) Effects of thyroid hormone on cardiac function: the relative importance of heart rate, loading conditions, and myocardial contractility in the regulation of cardiac performance in human hyperthyroidism. J Clin Endocrinol Metab 87:968-974

9. Biondi B, Palmieri EA, Fazio S et al (2000) Endogenous subclinical hyperthyroidism affects quality of life and cardiac morphology and function in young and middle-aged patients. J Clin Endocrinol Metab 85:4701-4705

10. Abonowara A, Quraishi A, Sapp JL et al (2012) Prevalence of atrial fibrillation in patients taking TSH suppression therapy for management of thyroid cancer. Clin Invest Med 35:E152-E156

11. Abdulrahman RM, Delgado V, Hoftijzer HC et al (2011) Both exogenous subclinical hyperthyroidism and short-term overt hypothyroidism affect myocardial strain in patients with differentiated thyroid carcinoma. Thyroid 21:471-476

12. Smit JW, Eustatia-Rutten CF, Corssmit EP et al (2005) Reversible diastolic dysfunction after long-term exogenous subclinical hyperthyroidism: a randomized, placebo-controlled study. J Clin Endocrinol Metab 90:6041-6047

13. Shargorodsky M, Serov S, Gavish D et al (2006) Long-term thyrotropin-suppressive therapy with levothyroxine impairs small and large artery elasticity and increases left ventricular mass in patients with thyroid carcinoma. Thyroid 16:381-386

14. Horne MK 3rd, Singh KK, Rosenfeld KG et al (2004) Is thyroid hormone suppression therapy prothrombotic? J Clin Endocrinol Metab 89:4469-4473

15. Volzke H, Robinson DM, Schminke U et al (2004) Thyroid function and carotid wall thickness. J Clin Endocrinol Metab 89:2145-2149

16. Biondi B, Cooper DS (2010) Benefits of thyrotropin suppression versus the risks of adverse effects in differentiated thyroid cancer. Thyroid 20:135-146

17. Klein Hesselink EN, Lefrandt JD, Schuurmans EP et al (2015) Increased risk of atrial fibrillation after treatment for differentiated thyroid carcinoma. J Clin Endocrinol Metab 100:4563-4569

18. Pajamaki N, Metso S, Hakala T et al (2018) Long-term cardiovascular morbidity and mortality in patients treated for differentiated thyroid cancer. Clin Endocrinol (Oxf) 88:303-310

19. Zoltek M, Nordenvall C, Ihre-Lundgren C et al (2017) Cardiovascular mortality in 6900 patients with differentiated thyroid cancer: a Swedish population-based study. Clin Surg 2:1519

20. Ludvigsson JF, Otterblad-Olausson P, Pettersson BU et al (2009) The Swedish personal identity number: possibilities and pitfalls in healthcare and medical research. Eur J Epidemiol 24:659-667

21. Ludvigsson JF, Andersson E, Ekbom A et al (2011) External review and validation of the Swedish national inpatient register. BMC Public Health 11:450

Publisher's Note Springer Nature remains neutral with regard to jurisdictional claims in published maps and institutional affiliations. 